before the differentiation of thyrocytes and is suppressed when thyroglobulin begins to be expressed (13). What seems clear is that the identification of Foxi1's role in intercalated cell differentiation has opened the door to an exciting new chapter in the development of the kidney.

Address correspondence to: Qais AlAwqati, Departments of Medicine and of Physiology and Cellular Biophysics, College of Physicians and Surgeons, Columbia University, 630 West 168th Street, New York, New York 10032, USA. Phone: (212) 305-3512; Fax: (212) 305-3475; E-mail: qa1@columbia.edu.

1. Blomqvist, S.R., et al. 2004. Distal renal tubular acidosis in mice that lack the forkhead transcrip- tion factor Foxi1. J. Clin. Invest. 113:1560-1570. doi:10.1172/JCI200420665.

2. Schwartz, G.J., Barasch, J., and Al-Awqati, Q. 1985. Plasticity of functional epithelial polarity. Nature. 318:368-371.

3. Schwartz, G.J., et al. 2002. Acid incubation reverses the polarity of intercalated cell transporters, an effect mediated by hensin. J. Clin. Invest. 109:89-99. doi:10.1172/JCI200213292.

4. Vijayakumar, S., Takito, J., Hikita, C., and Al-Awqati, Q. 1999. Hensin remodels the apical cytoskeleton and induces columnarization of intercalated epithelial cells: processes that resemble terminal differentiation. J. Cell Biol. 144:1057-1067.

5. Evan, A.P., Satlin, L.M., Gattone, V.H., 2nd, Connors, B., and Schwartz, G.J. 1991. Postnatal maturation of rabbit renal collecting duct. II. Morphological observations. Am. J. Physiol. 261:F91-F107.

6. Satlin, L.M., and Schwartz, G.J. 1987. Postnatal maturation of rabbit renal collecting duct: intercalated cell function. Am. J. Physiol. 253:F622-F635.

7. Aigner, J., Kloth, S., Jennings, M.L., and Minuth, W.W. 1995. Transitional differentiation patterns of principal and intercalated cells during renal collect- ing duct development. Epithelial Cell Biol. 4:121-130. 8. Hulander, M., et al. 2003. Lack of pendrin expression leads to deafness and expansion of the endolymphatic compartment in inner ears of Foxi1 null mutant mice. Development. 130:2013-2025.

9. Lehmann, O.J., Sowden, J.C., Carlsson, P., Jordan, T., and Bhattacharya, S.S. 2003. Fox's in development and disease. Trends Genet. 19:339-344.

10. Gaudet, J., and Mango, S.E. 2002. Regulation of organogenesis by the Caenorhabditis elegans FoxA protein PHA-4. Science. 295:821-825.

11. Overdier, D.G., Ye, H., Peterson, R.S., Clevidence, D.E., and Costa, R.H. 1997. The winged helix transcriptional activator HFH-3 is expressed in the distal tubules of embryonic and adult mouse kidney. J. Biol. Chem. 272:13725-13730.

12. Clifton-Bligh, R.J., et al. 1998. Mutation of the gene encoding human TTF-2 associated with thyroid agenesis, cleft palate and choanal atresia. Nat. Genet. 19:399-401.

13. Zannini, M., et al. 1997. TTF-2, a new forkhead protein, shows a temporal expression in the developing thyroid which is consistent with a role in controlling the onset of differentiation. EMBOJ. 16:3185-3197.

\title{
The case of visceral fat: argument for the defense
}

\section{Samuel Klein}

Center for Human Nutrition and Division of Geriatrics and Nutritional Sciences, Washington University School of Medicine, St. Louis, Missouri, USA.

\begin{abstract}
Increased plasma fatty acid concentrations may be responsible for many of the metabolic abnormalities associated with abdominal obesity. Excessive visceral fat is associated with insulin resistance and other metabolic risk factors for coronary heart disease. A study reported in this issue of the JCI evaluates the relative contribution of fatty acids released during lipolysis of visceral adipose tissue triglycerides to portal and systemic fatty acid flux in human subjects (see the related article beginning on page 1582).
\end{abstract}

The relationship between excess abdominal fat mass and insulin resistance was recognized a half century ago, when Jean Vague, a French physician, reported an association between a "masculine" or "android" obesity phenotype and diabetes (1). Subsequently, many large epidemiological and smaller physiological studies have confirmed the relationship between abdominal obesity and insulin resistance, diabetes, and other metabolic risk factors for coronary heart disease (2-5). In fact, excess abdominal fat is even associated with impaired insulin-mediated glucose uptake in lean adults (6).

Abdominal fat is composed of several distinct anatomic depots: subcutaneous fat, which can be divided into anterior and posterior or superficial and deep layers, and intraabdominal fat, which can be divided

Conflict of interest: The author has declared that no conflict of interest exists.

Citation for this article: J. Clin. Invest. 113:1530-1532 (2004). doi:10.1172/JCI200422028. into intraperitoneal and retroperitoneal sites. Intraperitoneal fat, also known as visceral fat, is composed of mesenteric and omental fat masses. Although the absolute amount of each of these depots is much larger in upper-body obese than in lean persons, the relative amount of abdominal fat with respect to total body fat mass is often similar in both groups. For example, visceral fat constitutes about $10 \%$ of total body fat mass in lean and obese men (7).

The close relationship between abdominal fat (i.e., total, subcutaneous, and/or visceral fat) and metabolic disease has stimulated a clinical interest in identifying high-risk patients. Waist circumference is often used as a surrogate marker of abdominal fat because it correlates closely with total abdominal fat mass measured by computed tomography (8) and it is not practical to directly measure abdominal fat mass in a clinical setting. Based on data from epidemiological studies, the Expert Panel on the Identification, Evaluation, and Treatment of Overweight and
Obesity in Adults, convened by the NIH, proposed that men with a waist circumference greater than $102 \mathrm{~cm}$ (40 in.) and women with a waist circumference greater than $88 \mathrm{~cm}$ (35 in.) are at increased risk for metabolic diseases (9).

\section{Fatty acid metabolism and insulin resistance}

The association between abdominal fat and insulin resistance does not prove causality, and it is possible that environmental, biological, or inherited factors that induce insulin resistance also cause abdominal fat accumulation (10). Nonetheless, it has been proposed that alterations in fatty acid metabolism associated with abdominal obesity are responsible for impaired insulin action because excessive circulating FFAs inhibit the ability of insulin to stimulate muscle glucose uptake and to suppress hepatic glucose production (11). The notion of a link between abdominal fat, FFA metabolism, and insulin resistance is supported by the observation that basal wholebody FFA flux rates are greater in upperbody obese than in lower-body obese and lean subjects $(12,13)$ and that diet-induced weight loss decreases whole-body FFA flux and improves insulin sensitivity (14). It has been hypothesized that excess visceral fat is more harmful than excess subcutaneous fat, because lipolysis of visceral adipose tis- 


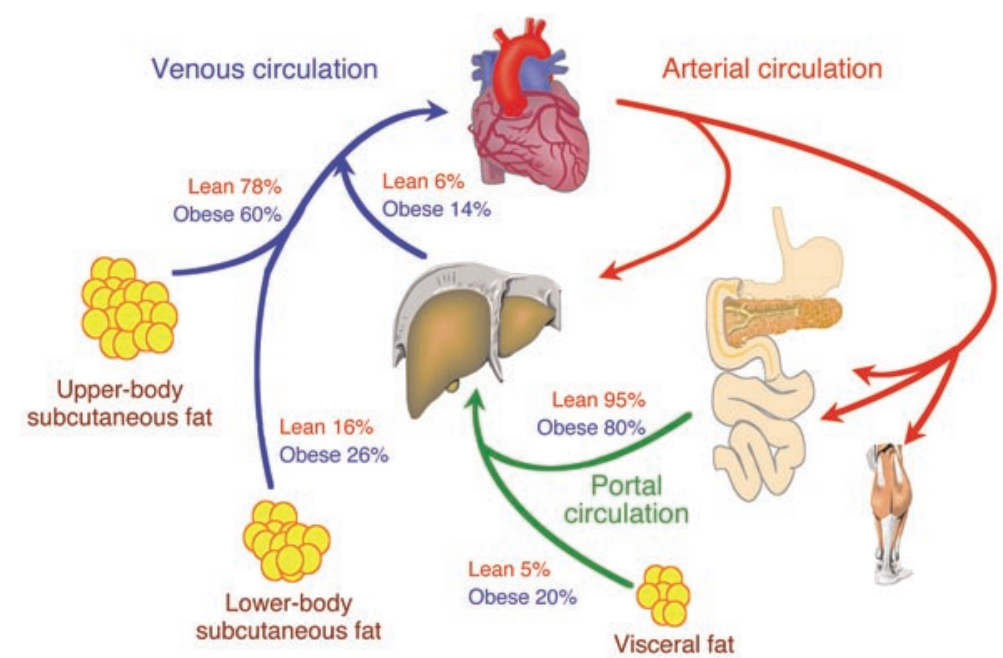

Figure 1

Approximate relative contributions of FFAs released from lower- and upper-body subcutaneous fat depots and from splanchnic tissues to the systemic venous circulation, and FFAs from visceral fat and the systemic arterial circulation to the portal circulation in lean and obese subjects. Values are based on data from ref. 20.

sue triglycerides releases FFAs into the portal vein, which are then delivered directly to the liver (15).

The precise relationship between individual abdominal fat depots and insulin resistance is not clear, because of conflicting results from different studies. Data from studies that evaluated insulin sensitivity, by using the "gold standard" euglycemichyperinsulinemic clamp technique, found that insulin-mediated glucose disposal (i.e., muscle insulin sensitivity) was inversely associated with visceral fat mass, abdominal subcutaneous fat, or both (7, 16-19), and that insulin-mediated suppression of glucose production (i.e., hepatic insulin sensitivity) was inversely proportional to both visceral fat and abdominal subcutaneous fat (16). Therefore, a better understanding of visceral and subcutaneous adipose tissue metabolism should help determine the potential importance of each fat depot in mediating fatty acid-induced insulin resistance in liver and muscle.

\section{Portal and systemic fatty acid kinetics}

In this issue of the JCI, Nielsen and colleagues report the results of a study that sheds new light on portal and systemic fatty acid kinetics in human subjects (20). By using sophisticated tracer methods in conjunction with mathematical modeling and technically demanding catheterization procedures, these investigators evaluated regional leg and splanchnic (intestine, spleen, pancreas, liver, and visceral fat) FFA metabolism and were able to determine the relative contributions of FFAs released from visceral fat into the portal and systemic circulations in lean and obese men and women (summarized in Figure 1).

The results of this study demonstrate that the release of FFAs into the portal vein from lipolysis in visceral fat depots increases with increasing amounts of fat (20). However, the relative contribution at any individual visceral fat mass was quite variable. For example, in subjects with approximately $150 \mathrm{~cm}^{2}$ of visceral fat, the percentage of total FFAs delivered to the liver that were derived from visceral fat ranged from $0 \%$ to $45 \%$, and the relative contribution of FFAs from visceral fat was lower in some subjects with a larger amount of visceral fat (about $300 \mathrm{~cm}^{2}$ ) than it was in others with a small amount (about $10 \mathrm{~cm}^{2}$ ). Therefore, although there is a direct relationship between visceral fat mass and its contribution to hepatic FFA metabolism, it is impossible to determine which individuals have a high rate of visceral FFA flux based on analysis of body composition and fat distribution alone. More importantly, the relative amount of portal vein FFAs derived from visceral fat was much less than the relative amount derived from lipolysis of subcutaneous fat. Subcutaneous adipose tissue depots release fatty acids into the venous circulation, which are then transported to splanchnic tissues by the arterial circulation. Only $5-10 \%$ of these fatty acids are metabolized by nonhe- patic splanchnic tissues, and the rest enter the portal vein (21). Nielson and colleagues found that only approximately 5\% and 20\% of portal vein FFAs originated from visceral fat in lean and obese subjects, respectively (Figure 1). The effect these additional fatty acids may have on insulin action in the liver is not known, but these data demonstrate that visceral fat is not as important as subcutaneous fat in supplying FFAs to the liver in lean or in most obese persons.

If fatty acids released from visceral fat contribute to insulin resistance in skeletal muscle, these FFAs must escape metabolism by the liver and enter the systemic circulation. Nielsen and colleagues found that only about $6 \%$ and $14 \%$ of the total FFAs that appear in the systemic circulation in lean and obese subjects, respectively, enter from the hepatic veins draining the liver (Figure 1) (20). Moreover, most of the fatty acids that pass through the liver are derived from lipolysis of subcutaneous fat, which releases FFAs that are ultimately delivered to the liver through the portal vein (about $80 \%$ of hepatic blood flow) and the hepatic artery (about $20 \%$ of hepatic blood flow). Therefore, very few fatty acids released from visceral fat itself are ever seen by skeletal muscle in either lean or obese individuals.

\section{Clinical implications and future directions}

The results of the study by Nielsen and colleagues (20) demonstrate that the contribution of FFAs derived from visceral fat to the portal and systemic circulations increases with increasing visceral fat mass. In some obese persons, fatty acid release from visceral adipose tissue triglycerides is substantial and could be an important factor in developing hepatic insulin resistance. However, excessive fatty acid release from visceral fat is unlikely to be a major factor in the pathogenesis of insulin resistance in skeletal muscle because it represents a very small percentage of total FFAs delivered to muscle tissues.

This study provides an important framework for future research. Additional studies are needed to determine the relationship between FFA delivery to the liver and hepatic insulin sensitivity, the implications of visceral fat metabolism during postprandial conditions, the factors responsible for intersubject variability in the contribution of visceral fat to total FFAs delivered to the liver, the impact of proteins and cytokines secreted by visceral and subcutaneous fat on hepatic and muscle insulin sensitivity, and the relationship between visceral fat and ectopic fat 
distribution in liver and muscle cells, which can also influence insulin action.

Address correspondence to: Samuel Klein, Washington University School of Medicine, 660 South Euclid Avenue, Campus Box 8031, St. Louis, Missouri 63110, USA. Phone: (314) 362-8699; Fax: (314) 362-8230.

1. Vague, J. 1956. The degree of masculine differentiation of obesities: a factor determining predisposition to diabetes, atherosclerosis, gout, and uric calculous disease. Am. J. Clin. Nutr. 4:20-34.

2. Kissebah, A.H., et al. 1982. Relation of body fat distribution to metabolic complications of obesity. J. Clin. Endocrinol. Metab. 54:254-260.

3. Folsom, A.R., et al. 2000. Associations of general and abdominal obesity with multiple health outcomes in older women. Arch. Intern. Med. 160:2117-2128.

4. Chan, J.M., et al. 1994. Obesity, fat distribution, and weight gain as risk factors for clinical diabetes in men. Diabetes Care. 17:961-969.

5. Poulliot, M.-C., et al. 1992. Visceral obesity in men. Diabetes. 41:826-834.

6. Ruderman, N., Chisholm, D., Pi-Sunyer, X., and Schneider, S. 1998. The metabolically obese, normalweight individual revisited. Diabetes. 47:699-713.
7. Abate, N., Burns, D., Peshock, R.M., Stray-Gundersen, J., and Grundy, S.M. 1995. Relationship of generalized and regional adiposity to insulin sensitivity in men. J. Clin. Invest. 96:88-98.

8. Pouliot, M.C., et al. 1994. Waist circumference and abdominal sagital diameter: best simple anthropometric indexes of abdominal visceral adipose tissue accumulation and related cardiovascular risk in men and women. Am. J. Cardiol. 73:460-468.

9. National Institutes of Health. 1998. Clinical Guidelines on the Identification, Evaluation, and Treatment of Overweight and Obesity in Adults: the Evidence Report [erratum 1998, 6:464]. Obes. Res. 6(Suppl. 2):51S-209S

10. Frayn, K.N. 2000. Visceral fat and insulin resistance: causative or correlative? Br. J. Nutr. 83(Suppl. 1):S71-S77.

11. Boden, G. 1997. Role of fatty acids in the pathogenesis of insulin resistance and NIDDM. Diabetes. 46:3-10.

12. Horowitz, J.F., Coppack, S.C., Paramore, D., Cryer, P.E., and Klein, S. 1999. Effect of short-term fasting on lipid kinetics in lean and obese women. Am.J. Physiol. 276:E278-E284.

13. Jensen, M.D., Haymond, M.W., Rizza, R.A., Cryer, P.E., and Miles, J.M. 1989. Influence of body fat distribution on free-fatty acid metabolism in obesity. J. Clin. Invest. 80:1168-1173.

14. Klein, S., Luu, K., Gasic, S., and Green, A. 1996. Effect of weight loss on whole-body and cellular lipid metabolism in severely obese humans. Am.J. Physiol. 270:E739-E745

15. Bjorntorp, P. 1990. Portal adipose tissue as a generator of risk factors for cardiovascular disease and diabetes. Arteriosclerosis. 10:493-496.

16. Abate, N., et al. 1996. Relationship of generalized and regional adiposity to insulin sensitivity in men with NIDDM. Diabetes. 45:1684-1693.

17. Goodpaster, B.H., Thaete, F.L., Simoneau, J., and Kelley, D.E. 1997. Subcutaneous abdominal fat and thigh muscle composition predict insulin sensitivity independently of visceral fat. Diabetes. 46:1579-1585.

18. Banerji, M.A., Chaiken, R.L., Gordon, D., Kral, J.G., and Lebovitz, H.E. 1995. Does intra-abdominal adipose tissue in black men determine whether NIDDM is insulin-resistant or insulin-sensitive? Diabetes. 44:141-145.

19. Colberg, S., Simoneau, J.-A., Thaete, F.L., and Kelley, D.E. 1995. Skeletal muscle utilization of free fatty acids in women with visceral obesity. J. Clin. Invest. 95:1846-1853.

20. Nielsen, S., Guo, Z.K., Johnson, C.M., Hensrud, D.D., and Jensen, M.D. 2004. Splanchnic lipolysis in human obesity. J. Clin. Invest. 113:1582-1588. doi: $10.1172 / \mathrm{JCI} 200421047$

21. Basso, L.V., and Havel, R.J. 1970. Hepatic metabolism of free fatty acids in normal and diabetic dogs. J. Clin. Invest. 49:537-547.

\section{Getting stents to go with the flow}

\section{R. Wayne Alexander}

Department of Medicine, Division of Cardiology, Emory University School of Medicine, Atlanta, Georgia, USA.

\begin{abstract}
Implantation of expandable stents into stenotic arteries after percutaneous coronary intervention to relieve arterial narrowing has become a standard therapeutic tool. The improvement in vascular interventional technology, and especially stent technology, has, arguably, outstripped understanding of the biologic consequences of opening an obstructed artery. In the case of bifurcation stenoses, new evidence suggests that opening a stenotic subsidiary branch may create unfavorable hemodynamics in the stented main branch that can lead to in-stent restenosis (see the related article beginning on page 1607).
\end{abstract}

The branching arterial system has complex hemodynamics. Flow is laminar in straight segments away from the ostia of side branches or the flow dividers that form the origin of subsidiary vessels of a main branch. The inherently disturbed flow at branch points creates an environment that predisposes to the development of atherosclerosis $(1,2)$. This permissive environment is characterized by low, oscillating, or reversed flow created opposite flow dividers or branch points and is caused by flow separation in which streamlines of flowing blood curve away from the artery wall

Conflict of interest: The author has declared that no conflict of interest exists.

Citation for this article: J. Clin. Invest. 113:1532-1534 (2004). doi:10.1172/JCI200422000. proximally and back toward the wall distally (3). The impact of local hemodynamic influences on the pathogenesis of atherosclerosis has been studied extensively, both in vitro and in vivo (4). In vitro flow models have defined the effects of a given geometry on flow dynamics, and biologic responses have been associated with predicted flow patterns $(5,6)$. Generally, these models have not incorporated consideration of the evolving effects of the dynamic outward or inward remodeling of arteries that is associated with atherosclerosis (7). In particular, such approaches have not been applied extensively to exploration of the consequences of the acute hemodynamic changes inherent in the practice of interventional cardiology for clinical outcomes, specifically restenosis. The optimum approach to the clinical situation of, for example, the presence of proximal stenoses in both the main branch and a relatively large side branch of a coronary artery has not been agreed upon generally (8). With the advent of the use of vascular stents, a common approach has been to stent both arteries, although longterm outcomes are less than optimal (9). A guiding clinical principle has been the drive for complete revascularization. The underlying vascular biology and, in particular, the interdependence of the hemodynamic environment in the main and side branches in the presence of stenoses in each has been poorly understood. Thus, beyond the imperative of relieving ischemia, there has been no firm biologic basis guiding clinical decision-making with regard to intervention to open a severely stenotic side branch when percutaneous coronary intervention is being performed to open the main branch stenosis.

\section{In vitro simulation of flow patterns of branching arteries}

In this issue of the JCI, Richter and colleagues describe experimental approaches that represent a major advance in understanding the interrelatedness of stenoses 